\title{
Análisis de la docencia en línea en el proceso enseñanza-aprendizaje: efectos colaterales del SARS-COV2 en la asignatura Fundamentos Médicos del Máster Ingeniería Biomédica
}

\section{Dolores Ortiz-Masià}

Profesora Contratada Doctora, Departamento de Medicina. Facultad de Medicina y Odontología. Universitat de Valencia (m.dolores.ortiz@uv.es).

\section{\$ EWWDFW}

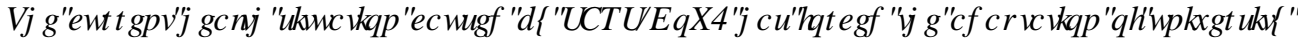

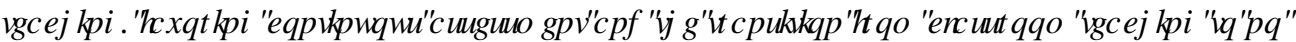

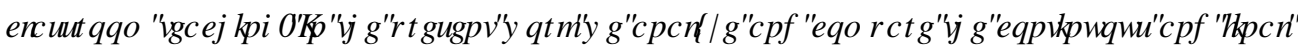

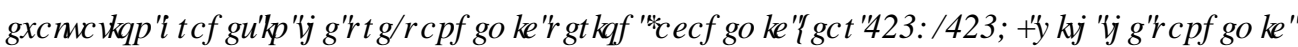

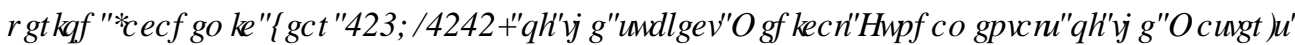

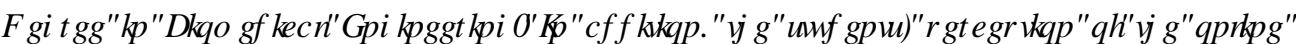

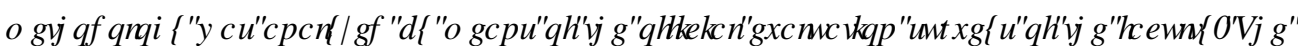

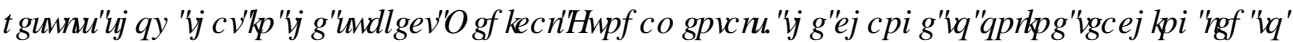

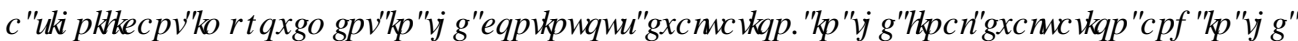

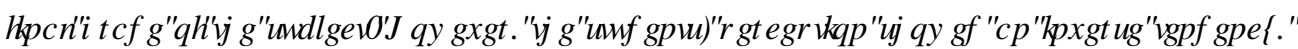

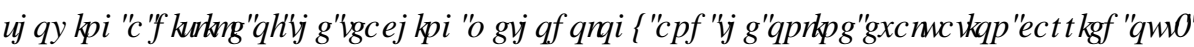

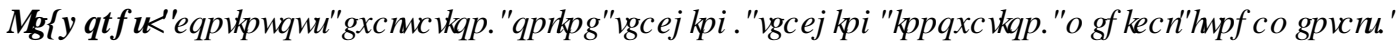
P DMAUVUWGHW

\section{HXP HQ}

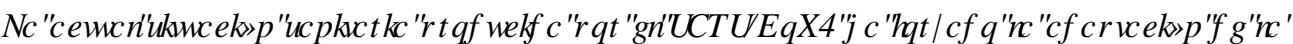

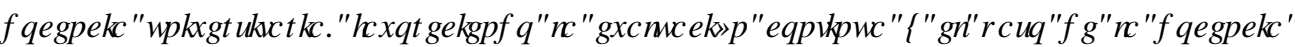

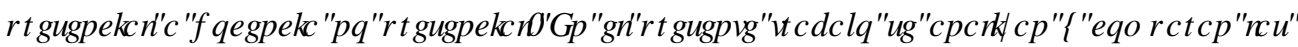

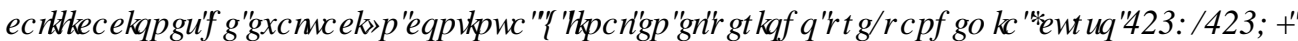

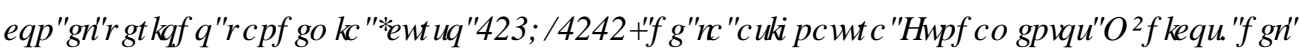

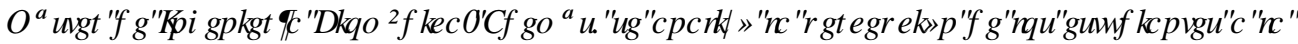

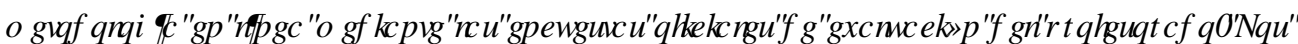

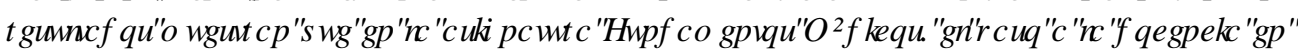

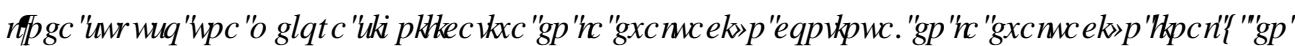

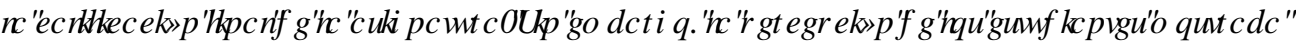

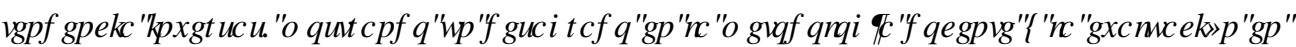
QQQHDUHDO] DOD]

3 DOEWVVFOYH P PGFRVIMUKGRVGHP i WHU 


\section{Introducción}

El 11 de marzo de 2020 la organización mundial de la Salud (OMS) declaró que el brote de coronavirus COVID-19 se había convertido en una pandemia global. Desde ese momento el mundo y las sociedades en cada país, han vivido una de las situaciones más críticas en la historia de la humanidad. Las condiciones de confinamiento forzoso, distanciamiento social y paralización de actividades, en prácticamente todas las naciones, han afectado severamente la vida cotidiana y las acciones de mujeres y hombres en todo el planeta.

La educación superior, no ha sido una excepción. A nivel global, nacional y local, el impacto es similar a otras actividades humanas y también asume formas particulares por las características específicas de las actividades docentes, de investigación y de extensión de este nivel educativo (Marinoni, Van't Land, \& Jensen, 2020). Según la UNESCO, unos 1.500 millones de estudiantes de 165 países no han podido asistir a los centros de enseñanza debido a la COVID-19. La pandemia ha obligado a la comunidad académica internacional a explorar nuevas formas de enseñar y aprender, incluida la educación a distancia y en línea. Esta situación ha resultado difícil tanto para los estudiantes como para los docentes (UNESCO).

Dos tercios de la enseñanza universitaria presencial ha sido sustituida por la enseñanza y el aprendizaje a distancia y una cuarta parte de las actividades universitarias están actualmente suspendidas pero la institución está trabajando en el desarrollo de soluciones para continuar con la enseñanza y el aprendizaje, a través de medios digitales o de autoaprendizaje (Marinoni, Van’t Land, \& Jensen, 2020).

En lo que concierne al rendimiento académico, la educación en línea ha demostrado que los resultados obtenidos son equivalentes a los que se obtienen en la educación presencial. Se han realizado estudios comparativos a nivel de educación media y educación superior y no se han encontrado diferencias significativas en los resultados obtenidos (Halsne, A. M. \& Gatta, L. A., 2002, Hogan, R., 1997). Existe poca información acerca de la percepción de los alumnos sobre si para ellos el paso a la docencia en línea ha supuesto una mejora, con respecto a la docencia presencial tradicional.

En la Universidad de Valencia, se instauró la docencia en línea de forma síncrona o asíncrona el 1 de abril de 2020, concretamente en la asignatura de Fundamentos médicos del Máster Ingeniería Biomédica, se continuó la docencia teórica y de seminarios a través de la plataforma Blackboard de forma síncrona.

\section{Objetivos}

El objetivo del presente trabajo es analizar y comparar las variaciones en las calificaciones en la asignatura Fundamentos Médicos del Máster Ingeniera Biomédica en los cursos académicos 2018-2019 (pre pandemia) y 2019-2020 (pandemia), con el fin de conocer la influencia de docencia en línea y exámenes en línea que ha forzado la pandemia SARS-COV2.

En relación a los objetivos del alumnado se busca conocer su percepción y aceptación de la docencia y evaluación en línea.

\section{Descripción}

Fundamentos Médicos es una asignatura que forma parte del módulo denominado Complementos formativos. Este módulo está diseñado para que alumnos no Graduados en Ingeniería Biomédica puedan acceder al Máster de Ingeniería Biomédica. La asignatura permite al alumno acceder a los conocimientos elementales de Medicina y Cirugía necesario para realizar el Máster, ya que esta asignatura es obligatoria para aquellos egresados en una Ingeniera diferente al Grado de Ingeniería Biomédica. Este módulo al ser obligatorio se encuentra en primer curso del Máster, se imparte durante el segundo semestre del curso académico y consta de 4.5 créditos ECTS. 
La evaluación continua en esta asignatura tiene un peso del 50\% de la nota final. La actividad de evaluación continua se realiza por grupos. Es un proyecto de investigación, donde se siguen los patrones de una revisión bibliográfica sistemática. Esta actividad se inicia en el Seminario diseñado para ello, donde se explican las bases de datos más importantes en ciencias de la salud ( 3 XEP HG 6FRSXV, etc.), y las páginas oficiales donde se muestran los índices de calidad de las revistas (: HERILFHOFHLRXLQDOFLWURQUSRUWJetc.).

La evaluación final tiene un valor del $50 \%$ de la nota final, se realiza en el mes de mayo y es un examen tipo test de 30 preguntas objetivas de respuesta múltiple sobre aspectos teóricos y prácticos. En la figura 1 se muestra los porcentajes de cada actividad que contribuye a la nota final del alumno.

Tanto el curso 2018-2019, como en el curso 2019-2020, se siguió la misma metodología docente, clases magistrales y seminarios. Siendo la única diferencia la presencialidad del profesor y los alumnos en un aula física. Las clases en línea se realizaron con la plataforma Blackboard, de forma síncrona con el horario aprobado en la Comisión de Máster. Algunos profesores que imparten la asignatura tienen amplia experiencia en la docencia en línea y el uso de herramientas digitales (T. Civera, $H W D O D 2020$ ).

Tanto el curso 2018-2019, como en el curso 2019-2020 habían matriculados 13 alumnos donde la participación y asistencia fue del $100 \%$ en ambos cursos, ya que, como se ha comentado, es una asignatura obligatoria para poder acceder al Máster para aquellos Ingenieros no graduados en Ingeniería Biomédica.

En el caso del examen final, en el curso presencial (2018-2019) se realizó en las instalaciones de la Facultad de Medicina en formato papel. En el curso 2019-2020 pasó a ser un examen en línea síncrono, a través de la plataforma Aula Virtual. En ambos cursos, el examen constaba de 32 preguntas de respuesta múltiple, con 5 posibles respuestas, donde sólo una era correcta. La duración del examen en ambos casos fue de 50 minutos. La única diferencia entre ambos exámenes fue que en el curso 2019-2020, al ser en línea, el acceso a la preguntas era secuencial, el orden de aparición de las cuestiones era aleatorio y no se podía navegar por el examen. Esta decisión se tomó con el fin de evitar engaños o trampas.

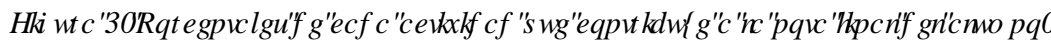

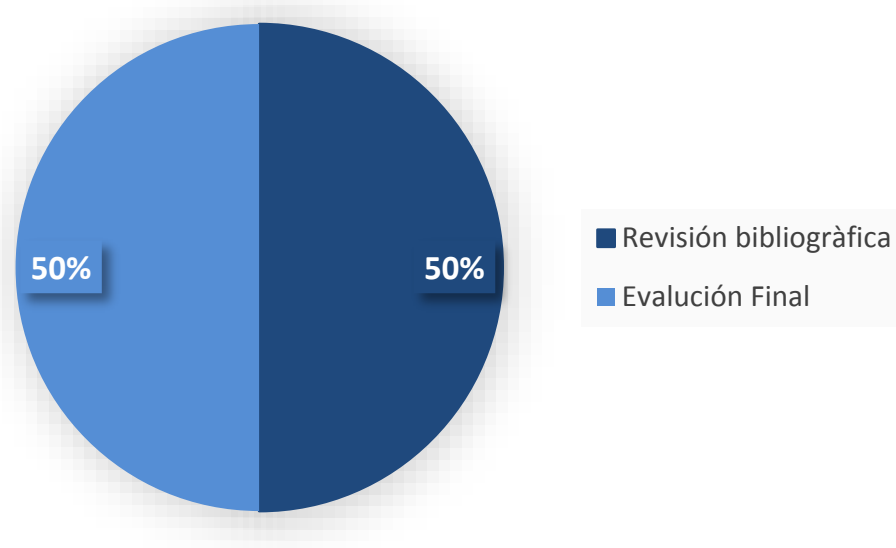

El cálculo de la calificación final se obtuvo de la media ponderada entre la calificación obtenida por la actividad revisión bibliográfica, con la obtenida en el examen final. Para el presente estudio no se tomaron cursos anteriores al 2018-2019 ya que la forma de evaluación era únicamente mediante evaluación final y el examen constaba de respuestas cortas. 
Para el estudio también se tomaron las encuestas de evaluación docente de la Universitat de Valencia de los cursos 2018-2019 y 2019-2020, con el fin de conocer la percepción de los alumnos. De todos los epígrafes se tomaron las evaluaciones que correspondían con los dos parámetros estudiados en este trabajo:

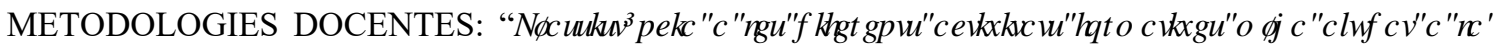

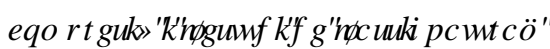

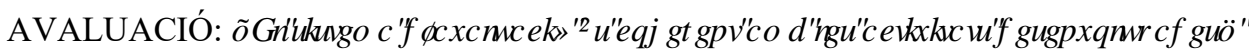

Estas afirmaciones tiene respuesta cerrada que va de 1 a 5 donde 1 (Escala Likert) ${ }^{3} 0$ RQWHQGHDFRLG y $5 \square$

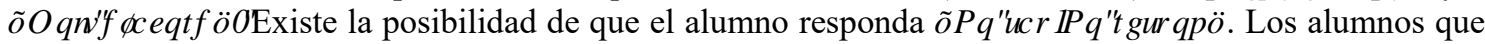
eligieron esta opción, para el estudio se tomó como pregunta no respondida.

La cumplimentación de las encuestas de evaluación docente es voluntaria y en línea. En el caso del curso 2018-2019, fue cumplimentada por el 100\% del alumnado. En el curso 2019-2020, únicamente cumplimentaron la encuesta 10 alumnos, que representa un 76,9\% de participación. Además a la afirmación

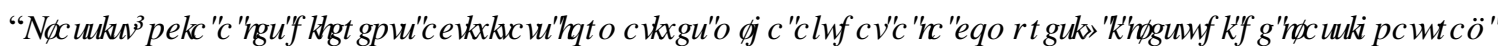
hubo dos alumnos que respondieron ${ }^{3} 1$ RाWS $\square$ R RUHSRQ $\llbracket$ por lo que el tamaño muestral se redujo a 8 , que supone una participación del $61,5 \%$.

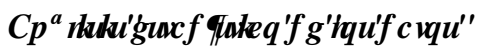

Los datos fueron expresados como media \pm Error Estándar de la Media (EEM) del grupo ( $\mathrm{n} \geq 8$ en todos los grupos) y fueron mediante la prueba $t$ de Student desapareada (Graph-Pad Sotware 6.0). Si el P-valor es $<0,05$ se consideran las diferencias entre hipótesis nula y alternativa como significativas.

\section{Resultados}

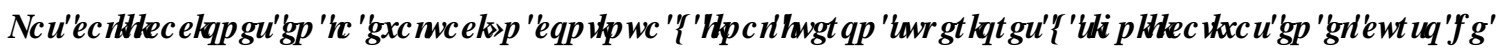 GRFHFIDHQIOQHUHSHFRDOFXURSUHOFIDO}

Se analizó la relación que había entre la calificación de la evaluación continua en el curso 2018-2019 (pre pandemia), donde el Seminario fue presencial, con las notas obtenidas en el curso 2019-2020, donde el Seminario se impartió en línea de forma síncrona. En la Figura 2a observamos esta comparativa. Como se observa, en general, la calificación continua (trabajo Revisión bibliográfica) para el curso 2018-2019 fue de 42,65 $\pm 1,325(\mathrm{~N}=13)$ sobre 50 puntos, mientras que para el curso 2019-2020 subió de forma significativa hasta 5 puntos $(47,67 \pm 0,5531(\mathrm{~N}=13), \mathrm{P}=0.019)$.

Del mismo modo, se compararon las notas obtenidas en el test final de ambos cursos. Como se puede observar, la docencia en línea síncrona mejoró las notas del examen final en 10 puntos significativos (curso 2018-2019: 33,07 \pm 1,451 (N=13); curso 2019-2020: 43,42 \pm 0,7418 (N=13), $\mathrm{P}<0.0001)$ (Figura 2b).

Por último se compararon las notas finales de la asignatura Fundamentos Médicos. Los resultados mostraron también la tendencia al alza y significativa del curso 2019-2020 respecto al curso 2018-2019, pasando de una media de 7,573 $\pm 0,2415(\mathrm{~N}=13)$ sobre 10 para el curso 2018-2019, a una media de 9,110 $\pm 0,1025(\mathrm{~N}=13, \mathrm{P}<0.0001)$ para el curso 2019-2020 (Figura 2c). 


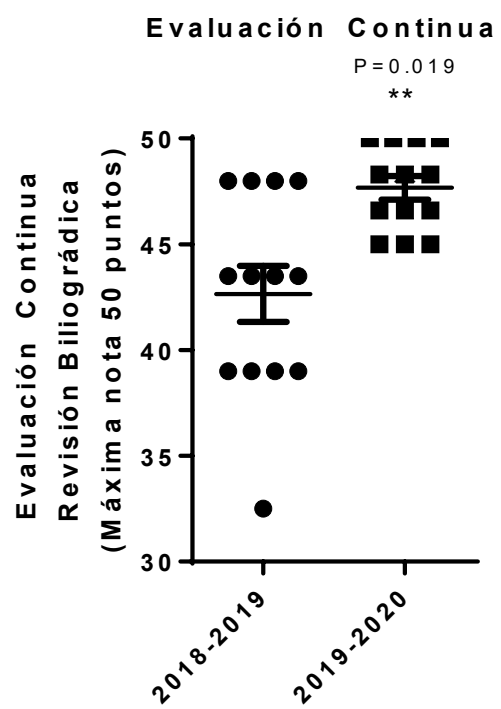

\begin{tabular}{|l|l|l|}
\hline & $2018-2019$ & $2019-2020$ \\
\hline Number of values & 13 & 13 \\
\hline & & \\
\hline Minimum & 32.50 & 45.00 \\
\hline $25 \%$ Percentile & 39.00 & 45.80 \\
\hline Median & 43.50 & 48.30 \\
\hline $75 \%$ Percentile & 48.00 & 50.00 \\
\hline Maximum & 48.00 & 50.00 \\
\hline & & \\
\hline Mean & 42.65 & 47.67 \\
\hline Std. Deviation & 4.776 & 1.994 \\
\hline Std. Error of Mean & 1.325 & 0.5531 \\
\hline & & \\
\hline Low er 95\% Cl of mean & 39.77 & 46.46 \\
\hline Upper 95\% Cl of mean & 45.54 & 48.87 \\
\hline \multicolumn{2}{|l}{} \\
\hline Sum & 554.5 & 619.7 \\
\hline
\end{tabular}

\begin{tabular}{|c|c|}
\hline \multicolumn{2}{|c|}{ Error Muestral } \\
\hline $\mathbf{2 0 1 8 - 2 0 1 9}$ & $\mathbf{2 0 1 9 - 2 0 2 0}$ \\
\hline 2,6 & 1,1 \\
\hline
\end{tabular}

$\mathrm{b}$

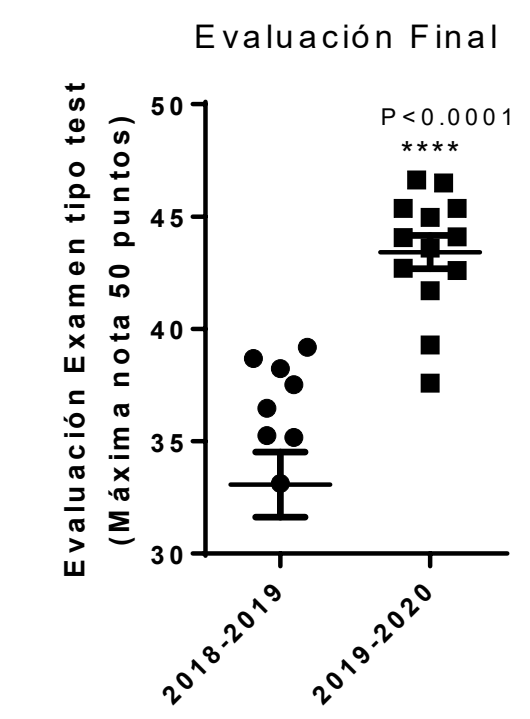

\begin{tabular}{|l|l|l|}
\hline & $2018-2019$ & $2019-2020$ \\
\hline Number of values & 13 & 13 \\
\hline Minimum & & \\
\hline 25\% Percentile & 23.28 & 37.58 \\
\hline Median & 28.36 & 42.15 \\
\hline $75 \%$ Percentile & 35.17 & 44.06 \\
\hline Maximum & 37.88 & 45.37 \\
\hline & 39.19 & 46.62 \\
\hline Mean & & \\
\hline Std. Deviation & 33.07 & 43.42 \\
\hline Std. Error of Mean & 5.233 & 2.675 \\
\hline & 1.451 & 0.7418 \\
\hline Low er 95\% Cl of mean & 29.91 & 41.80 \\
\hline Upper 95\% Cl of mean & 36.23 & 45.03 \\
\hline & & \\
\hline Sum & 429.9 & 564.4 \\
\hline
\end{tabular}

\begin{tabular}{|c|c|}
\hline \multicolumn{2}{|c|}{ Error Muestral } \\
\hline $\mathbf{2 0 1 8 - 2 0 1 9}$ & $\mathbf{2 0 1 9 - 2 0 2 0}$ \\
\hline 2,8 & 1,5 \\
\hline
\end{tabular}

(cc) EY-NC-ND 2021, Universitat Politècnica de València

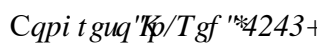


$\mathrm{c}$

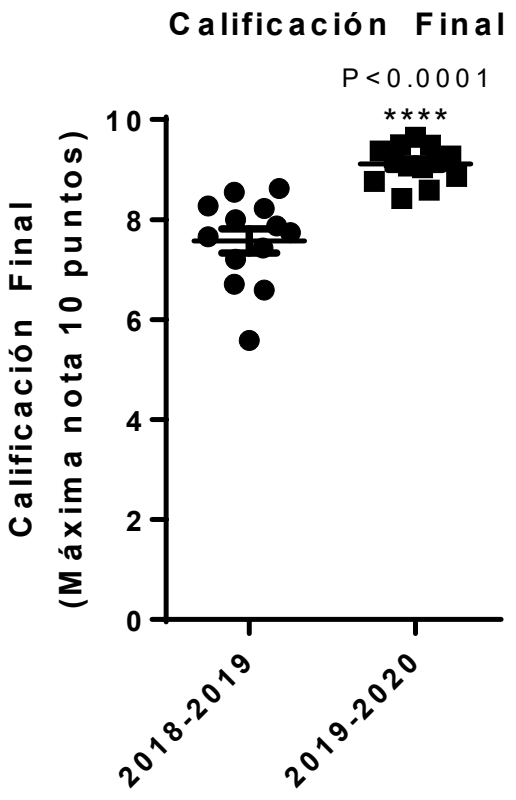

\begin{tabular}{|l|l|l|}
\hline & $2018-2019$ & $2019-2020$ \\
\hline Number of values & 13 & 13 \\
\hline & & \\
\hline Minimum & 5.580 & 8.420 \\
\hline 25\% Percentile & 6.960 & 8.810 \\
\hline Median & 7.740 & 9.170 \\
\hline $75 \%$ Percentile & 8.245 & 9.430 \\
\hline Maximum & 8.620 & 9.650 \\
\hline & & \\
\hline Mean & 7.573 & 9.110 \\
\hline Std. Deviation & 0.8707 & 0.3697 \\
\hline Std. Error of Mean & 0.2415 & 0.1025 \\
\hline & & \\
\hline Low er 95\% Cl of mean & 7.047 & 8.887 \\
\hline Upper 95\% Cl of mean & 8.099 & 9.333 \\
\hline \multicolumn{2}{|l}{} \\
\hline Sum & 98.45 & 118.4 \\
\hline
\end{tabular}

\section{Error Muestral}

\begin{tabular}{|c|c|}
\hline 2018-2019 & 2019-2020 \\
\hline 0.5 & 0.2 \\
\hline
\end{tabular}

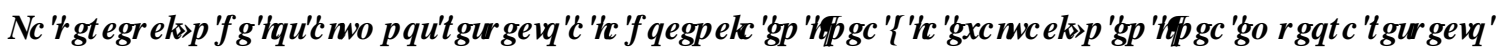 DOOSUHMPFIDO}

A continuación, se analizó la percepción de los estudiantes en relación a la metodología docente y al sistema de evaluación en la pandemia. Para ello se compararon las encuestas de evaluación docente de los cursos 2018-2019 donde la docencia y la evaluación fue totalmente presencial, con la del curso 2019-2020 donde tanto la docencia como la evaluación fueron completamente en línea. Como se puede observar en la Figura 3, existe una diferencia significativa, a la baja, entre ambos cursos. Esta diferencia se mostraba en la

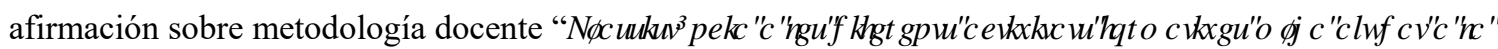

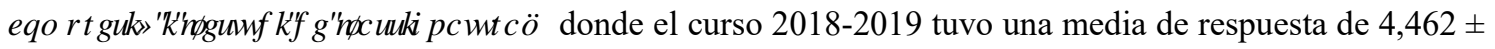
0,1831 ( $\mathrm{N}=13)$ sobre 5, respecto al curso 2019-2020, que bajó de forma importante a 2,500 $\pm 0,3727(\mathrm{~N}=10$, $\mathrm{P}<0.0001$ ) puntos sobre 5 (Figura $3 \mathrm{a}$ ).

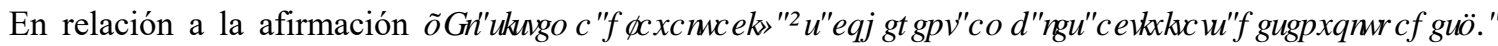
también mostró una reducción considerable pasando de 4,615 $\pm 0,1804(\mathrm{~N}=13)$ sobre 5 para el curso 20182019, a 2,625 $\pm 0,3239(\mathrm{~N}=8, \mathrm{P}<0.0001)$ puntos sobre 5, para el curso 2019-2020 (Figura 3b).

Todo ello nos informa que de forma general, que el cambio a metodologías digitales empeoró la apreciación de los estudiantes tanto para la docencia teórica como la evaluación en línea. 


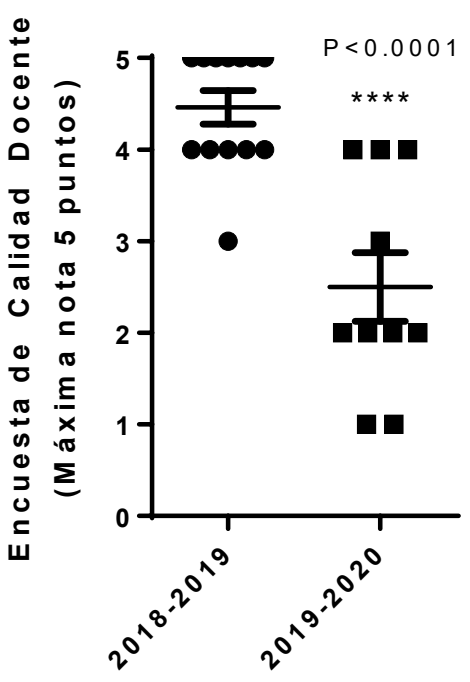

\begin{tabular}{|l|l|l|}
\hline & $2018-2019$ & $2019-2020$ \\
\hline Number of values & 13 & 10 \\
\hline Minimum & & \\
\hline $25 \%$ Percentile & 3.000 & 1.000 \\
\hline Median & 4.000 & 1.750 \\
\hline $75 \%$ Percentile & 5.000 & 2.000 \\
\hline Maximum & 5.000 & 4.000 \\
\hline & 5.000 & 4.000 \\
\hline Mean & & \\
\hline Std. Deviation & 4.462 & 2.500 \\
\hline Std. Error of Mean & 0.6602 & 1.179 \\
\hline & 0.1831 & 0.3727 \\
\hline Lower 95\% Cl of mean & 4.063 & 1.657 \\
\hline Upper 95\% Cl of mean & 4.861 & 3.343 \\
\hline & & \\
\hline Sum & 58.00 & 25.00 \\
\hline
\end{tabular}

\begin{tabular}{|c|c|}
\hline \multicolumn{2}{|c|}{ Error Muestral } \\
\hline $\mathbf{2 0 1 8 - 2 0 1 9}$ & $\mathbf{2 0 1 9 - 2 0 2 0}$ \\
\hline 0.4 & 0.7 \\
\hline
\end{tabular}

His togram a

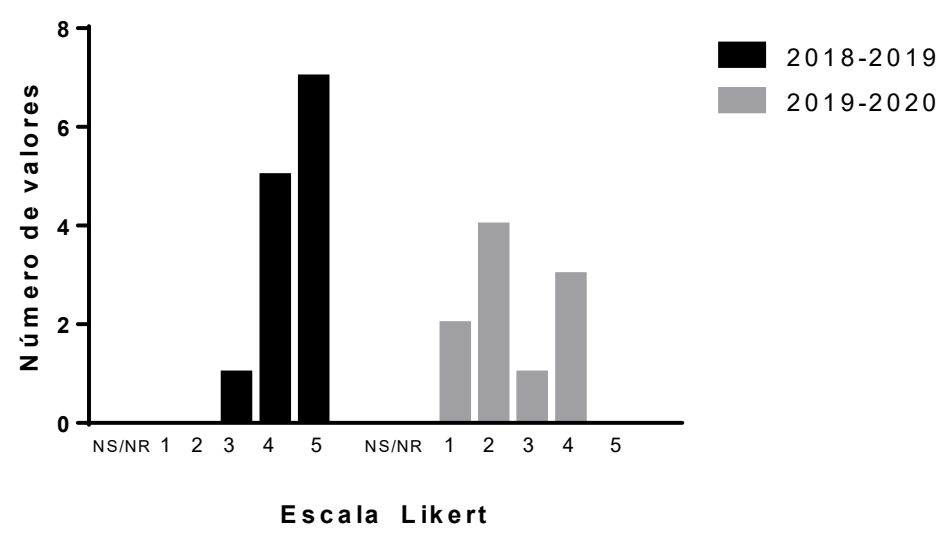


$L$ a asisten cia a las d iferentes activid ades form ativas $m$ e $h$ a resultado de ayuda para la com prensión y estudio de la asign atu ra

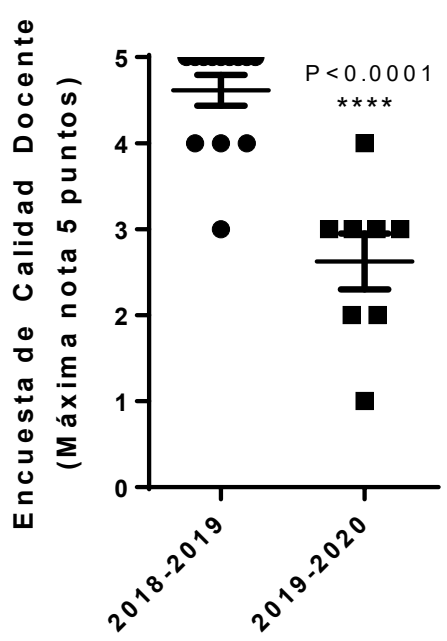

\begin{tabular}{|l|l|l|}
\hline & $2018-2019$ & $2019-2020$ \\
\hline Number of values & 13 & 8 \\
\hline & & \\
\hline Minimum & 3.000 & 1.000 \\
\hline $25 \%$ Percentile & 4.000 & 2.000 \\
\hline Median & 5.000 & 3.000 \\
\hline $75 \%$ Percentile & 5.000 & 3.000 \\
\hline Maximum & 5.000 & 4.000 \\
\hline & & \\
\hline Mean & 4.615 & 2.625 \\
\hline Std. Deviation & 0.6504 & 0.9161 \\
\hline Std. Error of Mean & 0.1804 & 0.3239 \\
\hline & & \\
\hline Lower 95\% Cl of mean & 4.222 & 1.859 \\
\hline Upper 95\% Cl of mean & 5.008 & 3.391 \\
\hline & & \\
\hline Sum & 60.00 & 21.00 \\
\hline
\end{tabular}

\begin{tabular}{|c|c|}
\hline \multicolumn{2}{|c|}{ Error Muestral } \\
\hline $\mathbf{2 0 1 8 - 2 0 1 9}$ & $\mathbf{2 0 1 9 - 2 0 2 0}$ \\
\hline 0.4 & 0.6 \\
\hline
\end{tabular}

Histogram a

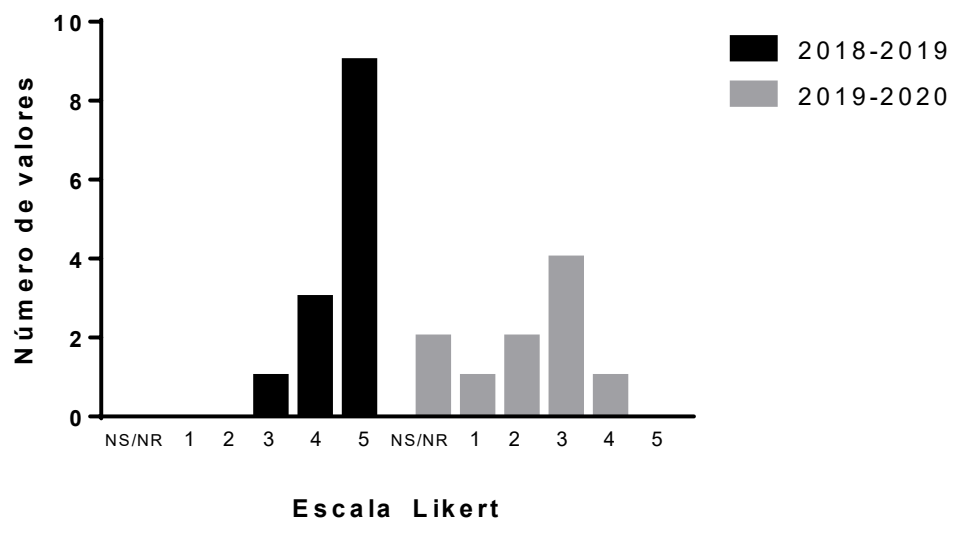




\title{
Conclusiones
}

Con el trabajo de investigación desarrollado en la presente memoria podemos concluir en primer lugar, que la docencia y evaluación en línea, realizada como consecuencia de la pandemia por SARS-COV2, mejoró el proceso enseñanza-aprendizaje de los alumnos. Se produjo un incremento en las puntuaciones globales de los alumnos, debido a mejoras sustanciales en las calificaciones de evaluación continua (revisión bibliográfica sistemática) y evaluación final (examen tipo test). Por otro lado, cabe añadir que esta mejora en el proceso enseñanza-aprendizaje no fue percibido de igual manera por los estudiantes donde evaluaron a la baja tanto la metodología en línea como la evaluación en línea, una posible razón del desagrado general en la metodología docente en línea podría ser por el hecho que al ser una asignatura médica es necesario muchas veces una interacción persona-persona para entender procedimientos y puntos clave de un proceso diagnóstico (localización del dolor, pruebas de fuerza muscular, etc.). En general, las clases magistrales de esta asignatura son eminentemente participativas, donde de forma asidua se detiene la clase para simular entre el profesor-estudiante una determinada exploración o un determinado síntoma. En la formación en línea, esta deficiencia se puede suplir con videos formativos, pero como muy bien describe la Taxonomía de Bloom (Bloom, B., Englehart, M. Furst, E., Hill, W., \& Krathwohl, 1956), la visualización de videos no desarrolla, de igual forma, el dominio afectivo del proceso de aprendizaje (vinculado al desarrollo de sentimientos y actitudes) ya que los estudiantes no aplican los conceptos que se están explicando. Todo ello desencadena que las clases en línea, en esta asignatura en concreto, se vuelvan tediosas y poco participativas, donde el estudiante pasa a ser un mero receptor de información, favoreciendo por tanto, una evaluación a la baja de la metodología docente en línea respecto a la presencial. Otro posible argumento, podría ser la habilidad del profesorado para transmitir la información y entusiasmo a través de una pantalla. Aunque el profesorado de esta asignatura tiene experiencia en el uso de herramientas digitales, no lo tiene en impartir docencia en línea, la cual requiere de un mayor esfuerzo para mantener la atención del estudiante ya que compite con un mayor número de parámetros de distracción (teléfonos, timbres, ruidos habituales en una vivienda) y los diálogos alumno-profesor son más rígidos.

Por otro lado, la peor impresión de los estudiantes en la evaluación en línea respecto a la presencial podría explicarse por diferentes motivos. Por ejemplo, por el hecho de que en la evaluación en línea los estudiantes se encontraban bajo mayor presión ya que las preguntas eran secuenciales, aleatorizadas y sin retorno. A diferencia del examen presencial, que con el mismo tiempo podían recorrer el examen con libertad. Aunque esta condición no afecta a la nota final de la asignatura, sí parece haber afectado a la buena percepción del alumnado.

Para finalizar y con el fin de resumir, concluimos que el presente trabajo avala el uso de herramientas docentes en línea ya que los resultados del proceso enseñanza-aprendizaje se mantienen, e incluso mejoran. Sin embargo, el uso de herramientas digitales podría limitar, a extremos no deseables por el alumnado, la conexión y el vínculo con el profesor, viéndose afectada el enlace con la asignatura y por tanto la ratificación por parte del alumnado.

\section{Agradecimientos}

\author{
Innovation Project Universitat de Valencia n ${ }^{\circ}$ UV-SFPIE_RMD17-586754. \\ Innovation Project Universitat de Valencia n ${ }^{\circ}$ UV-SFPIE_RMD18-841128. \\ Innovation Project Universitat de Valencia nº UV-SFPIE_PID19-1096660.
}




\section{Referencias}

\section{Artículo}

BLOOM, B., ENGLEHART, M. FURST, E., HILL, W., \& KRATHWOHL, D. (1956). 7DIRQRP।पRा

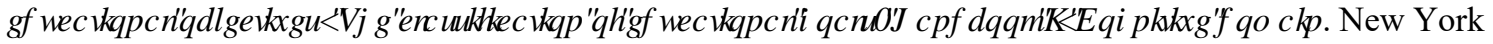
and Toronto: David McKay Co Inc.

HALSNE, A. M. \& GATTA, L. A., 2002. Online versus traditionally-delivered instruction: A descriptive study of learner characteristics in a community college setting. Online Journal of Distance Learning Administration, 5(1).

MARINONI, GIORGIO, VAN'T LAND, HILLIGJE, \& JENSEN, TRINE. (2020). 7KH, P SDFWRI\&2 9, '

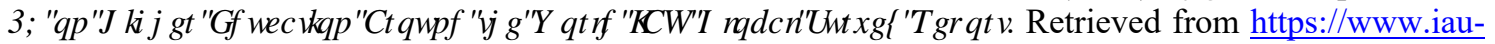
aiu.net/IMG/pdf/iau_covid19_and_he survey_report_final_may_2020.pdf

\section{Proceedings}

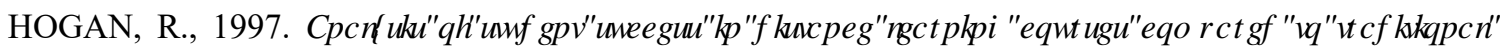
FRXUHMT6th Annual Conference on Multimedia in Education and Industry. Chattanooga, TN, EE. UU. (No. de servicio de reproducción de documentos ERIC No. ED 412 992)

T. CIVERA, M.D. BARRACHINA, S. CALATAYUD, S. MARTINEZ-HERVAS, A. ÁLVAREZ, D.

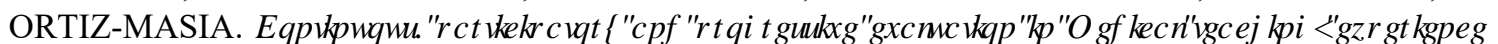

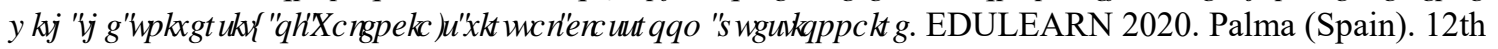
International Conference on education and new learning technologies. Conference Proceedings, 2020.

\section{Página WEB}

COVID-19 y educación superior: Aprender a desaprender para crear una educación para el futuro. https://www.un.org/es/impacto-acad\%C3\%A9mico/covid-19-y-educaci\%C3\%B3n-superior-aprenderdesaprender-para-crear-una-educaci\%C3\%B3n-para. Consultado el 29 de marzo de 2021. 